\title{
AFIDOFAUNA (HOM. APHIDIDAE) DE LA VERTIENTE SUR DE LA PROVINCIA FITOGEOGRÁFICA OROCANTÁBRICA, ESPAÑA
}

\author{
M.P. Mier Durante (*), A.K. Ghosh (**), H. Zaixso (***) \\ y J.M. Nieto Nafría $(*)$
}

\begin{abstract}
RESUMEN
La afidofauna de la vertiente sur de la Provincia fitogeográfica Orocantábrica (Región Eurosiberiana) está formada por 266 especies de pulgones (Hom. Aphididae), pertenecientes a nueve subfamilias. Se cita por primera vez en España Monaphis antennata y por segunda vez Acyrthosiphon echinospartii. Se citan otras 53 especies por vez primera en la zona. Se discute la distribución subfamiliar en relación con la fauna mundial. El sector fitogeográfico más rico en especies es el Ubiñense-Picoeuropeano. Existe un gradiente altitudinal en la distribución de las especies y parcialmente otro de este a oeste. Se discute la composición afidofaunística de las clases altitudinales más elevadas (desde $1.600 \mathrm{~m}$ ) y de la más baja de la Provincia (menos de $1.000 \mathrm{~m}$ ).
\end{abstract}

Palabras clave: Afidofauna, pulgones, Orocantábrica (Provincia fitogeográfica), Cordillera Cantábrica

\section{ABSTRACT \\ The aphidfauna (Hom. Aphididae) of the southern side of the Orocantabrian phytogeographic Province, Spain}

The Orocantabrian phytogeographic Province is situated in Cantabrian Mountain Range in the north-weast of the Iberian Peninsula. It is the only phytogeographic Province which is exclusively Iberian in the Eurosiberian phytogeographic Region. The aphidfauna of the southern side of the Province has been studied from bibliographic and unpublished data from 92 localities within the province of Leon. Two hundred and sixtysix species of aphids (Hom. Aphididae) belonging to 9 subfamilies are recorded. Monaphis antennata is reported for the first time in Spain and Acyrthosiphon echinospartii for the second. Another 53 species are recorded for the first time in the area. Family distribution with regard to world fauna is discussed. The distribution of aphid species in the three phytogeographic Sectors of the Province is discussed: the richest Sector is "Ubiñense-Picoeuropeano", probably because of its greater floristic diversity. The height distribution of the species is discussed, paying special attention to the absence of some species at lower altitudes (less that 1,000 m) and of others at higher altitudes $(1,600 \mathrm{~m}$ and higher). Taking into account phytogeographic-altitude inventories, a study of simalirity of the overall distribution of the aphids in the Province was carried out. The existence of an altitudinal gradient and up to a certain point of a longitudinal gradient (from east to west) is concluded.

Key words: Aphidfauna, aphids, Orocantabrian phytogeographic Province, Cantabrian Mountain Range

\section{Introducción}

Los pulgones (Hom. Aphididae) son insectos fitófagos, epífitos y de movilidad muy restringida, pues solamente las formas aladas emprenden vuelos en determinados momentos de su vida, siendo además considerablemente estrictos en su alimentación: monófagos u oligófagos (Dixon, 1985). Estas

\footnotetext{
* Departamento de Biología Animal. Universidad de León. 24071, León (España).

** Zoological Survey of India, New Alipore 'M' Block, Calcutta 700053 (West Bengal, India).

*** Facultad de Ciencias Naturales, Universidad Nacional de la Patagonia - San Juan Bosco. 9005 Comodoro Ribadavia (Argentina).
} 
características bionómicas determinan que los patrones de distribución de las diferentes especies de pulgones estén absolutamente condicionados por la distribución de las correspondientes plantas hospedadoras (Dixon, op. cit.). Como en otros animales, la distribución de las especies de pulgones está condicionada además por muchos otros factores, que no siempre conocemos o sabemos valorar ajustadamente. En resumen, teóricamente el área de distribución máxima de una especie de pulgón será el área conjunta de sus plantas hospedadoras, pudiendo ser menor, pero en ningún caso mayor.

Puesto que la distribución de las especies afidianas viene determinada por la de sus plantas hospedadoras, parece evidente que la delimitación fitogeográfica para realizar estudios y valoraciones afidofaunísticos ha de ser particularmente útil (Nieto Nafría et al., 1994). Con este trabajo damos a conocer y valoramos la afidofauna de la vertiente sur de la Provincia fitogeográfica Orocantábrica. rica de la Región Eurosiberiana (Rivas Martínez et al., 1984). Se extiende en una banda estrecha orientada de este a oeste, de unos $100 \mathrm{~km}$ de longitud, asentada sobre la Cordillera Cantábrica. Limita al norte con la Provincia Cantabro-atlántica, también eurosiberiana, y al sur con la Región Mediterránea (Rivas Martínez et al., op. cit.).

Debido a la proximidad al mar, las dos vertientes son muy diferentes. La vertiente norte desciende en algunas localidades hasta los $100 \mathrm{~m}$ y sus cotas bajas más elevadas no sobrepasan los $300 \mathrm{~m}$; mientras que la vertiente sur solamente desciende hasta los $800 \mathrm{~m}$ y normalmente hasta no más abajo de los $1.000 \mathrm{~m}$.

Según criterios fitogeográficos la Provincia se divide en tres sectores, que se suceden de este a oeste: Campurriano-Carrionés, UbiñensePicoeuropeano y Laciano-Ancarense. En el resto de este artículo (texto, tabla 1 y figura 1) los nombres de los sectores se abrevian: CC, UP, y LA, respectivamente.

\section{Zona de estudio}

La Provincia fitogeográfica Orocantábrica (figura 1) es la única Provincia exclusivamente ibé-

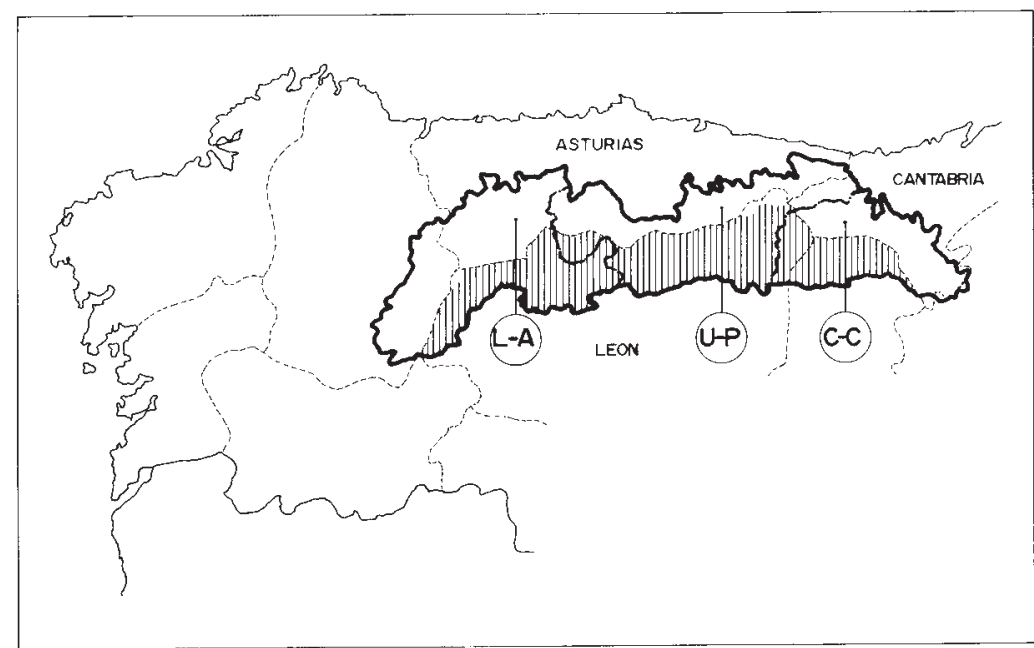

Fig. 1.- Mapa de la parte noroeste de la Península Ibérica con los límites de la Provincia fitogeográfica Orocantábrica y de sus tres sectores fitogeográficos (Campurriano-Carrionés, CC, Ubiñense-Picoeuropeano, UP, y Laciano-Ancarense, LA), señalándose la vertiente Sur de la Provincia. Dibujo de E. Ortega.

Fig. 1.- Map of the North-west part of the Iberian Peninsula with the limits of the Orocantabrian phytogeographic Province and its phytogeographic sectors (Campurriano-Carrionés, CC, Ubiñense-Picoeuropeano, UP, y Laciano-Ancarense, LA), showing the southern slope of the province. Illustration by E. Ortega. 


\section{Material y Métodos}

Para la preparación de la lista afidofaunística de la vertiente sur de la Provincia fitogeográfica Orocantábrica hemos utilizado dos tipos de datos: bibliográficos e inéditos.

Los datos bibliográficos son los aportados por los autores siguientes: 1) Robles Blanco y Mier Durante (1982), Gutiérrez Alaiz y Mier Durante (1983), López Rodríguez y Nieto Nafría (1983) y Robles García y Nieto Nafría (1983), que estudiaron la fauna leonesa de distintos grupos taxonómicos de pulgones. 2) Núñez Pérez (1992), que se centró en los de las plantas cultivadas en León. 3) Nieto Nafría et al. (1990 y 1991), Tizado Morales y Nieto Nafría (1991 y 1994) y Nieto Nafría y Mier Durante (1991), que trataron especies nuevas para la fauna ibérica o española. 4) Tizado Morales (1991), que llevó a cabo un pormenorizado estudio sobre los pulgones del valle del río Bernesga, aportando muchos datos a la afidofauna del sector UP.

Los datos inéditos proceden del trabajo de identificación realizado por dos de los autores (N.N. y M.D.) de los pulgones conservados en la colección afidológica del Departamento de Biología Animal de la Universidad de León. Han sido estudiadas cerca de 2.000 muestras, la mayoría de ellas recogidas por esos dos autores a lo largo de 15 años y en la campaña del verano de 1986 junto al Prof. G. Remaudière.

En su conjunto las muestras proceden de 92 localidades de la provincia de León, con altitudes comprendidas entre los $850 \mathrm{~m}$ y los $1.950 \mathrm{~m}$, situadas en los tres sectores fitogeográficos indicados (fig. 1): 13 en el sector CC, 64 en el Sector UP y 15 más en el Sector LA. La altura de cada localidad fue clasificada de acuerdo a 6 clases altitudinales: I, menos de 999 m; II, entre 1.000 y 1.199 m; III, entre 1.200 y $1.399 \mathrm{~m}$; IV, entre 1.400 y 1.599 ; V entre 1.600 y 1.799; y VI, entre 1.800 y 1.999. Con el fin de obtener una visión de conjunto de la afidofauna del sur de la Orocantábrica, evitando una presentación farragosa de los datos, aún a riesgo de disminuir la información, los datos se presentan agrupados por sectores y por clases altitudinales (ver tabla 1).

Para llevar a cabo el análisis de similitud, se establecieron inventarios fitoaltitudinales, estando constituido cada inventario por las especies que aparecen en un sector fitogeográfico y una clase de altura determinados. Se obtuvieron 15 inventarios (CC-II, CC-III, CC-IV y CC-V; UP-I, UP-II, UPIII, UP-IV, UP-V y UP-VI; LA-I, LA-II, LA-III, LA-IV y LA-VI), menos de los 18 previsibles, pues no había localidad alguna en las clases I y VI del sector CC, ni en la clase V del sector LA.

La similitud entre inventarios fue calculada mediante el índice de Sörensen (Legendre y Legendre, 1979). A partir de la matriz de similitud obtenida, se llevó a cabo el agrupamiento de los inventarios mediante el método UPGMA de aglomeración jerárquica o agrupamiento según la media aritmética (Sneath y Sokal, 1973; Legendre y Legendre, 1979). El número de grupos fue obtenido de acuerdo a la mayor distancia entre grupos en pasos sucesivos de aglomeración (Hair et al., 1992), siempre que el número de grupos formados fuera mayor de dos.

Se ha adoptado una ordenación taxonómica similar a la seguida por Dixon (1985) con el fin de facilitar las comparaciones. No obstante hemos considerado prudente segregar los pterocomatinos de la subfamilia Aphidinae y los telaxinos de la subfamilia Drepanosiphinae, considerándolos subfamilias independientes: Pterocommatinae y Thelaxinae, respectivamente.

Como el número de especies de varias de las subfamilias es muy bajo y en algunos casos sus citas muy pocas, los análisis por sectores y por clases altitudinales los hemos efectuado conjuntamente, sin considerar las subfamilias.

\section{Resultados y discusión}

\section{RESULTADOS GENERALES Y NOVEDADES FAUNÍSTICAS}

A partir de los datos disponibles, la afidofauna de la Provincia Orocantábrica está formada por 266 especies, presentes en localidades de sectores fitogeográficos y clases altitudinales según se muestra en la tabla 1, en la que se relacionan ordenadas alfabéticamente dentro de cada una de las subfamilias y de cada tribu en el caso de la subfamilia Aphidinae. La riqueza afidológica del Sur de la Provincia Orocantábrica queda puesta de manifies- 
to si consideramos que esas 266 especies son aproximadamente el $47 \%$ de la afidofauna española conocida, aún no habiendo ninguna especie endémica y presentando todas ellas una distribución más o menos amplia, al menos en Europa.

Monaphis antennata (conocida de Portugal, distrito de Viseu, según Ilharco, 1968) se cita por vez primera para España: La Robla, sobre Betula celtiberica; y Acyrthosiphon echinospartii se cita por primera vez después de su descripción (de la provincia de Orense en la Región fitogeográfica Mediterránea), sobre Genista sp., nueva planta hospedadora. Además de ellas, 53 especies se citan por vez primera (parte izquierda de la tabla 1: especies solamente marcadas con $\mathrm{N}$, una o varias veces) en el sur de la Orocantábrica: 5 penfiginos, 2 telaxinos, 7 drepanosifinos, 4 lacninos y 35 afidinos, con sólo 5 novedades entre los Aphidini.

\section{REPRESENTACIÓN SUBFAMILIAR}

La distribución subfamiliar de las 266 especies (11 Pemphiginae, 1 Anoeciinae, 1 Hormaphidinae, 3 Thelaxinae, 30 Drepanosiphinae, 11 Chaitophorinae, 13 Lachninae, 2 Pterocommatinae y 194 Aphidinae) está en líneas generales de acuerdo con la distribución general de las subfamilias en la fauna mundial (Dixon, 1985) y con la riqueza en especies de esas subfamilias en la fauna paleárticooccidental (Dixon, op. cit. y datos a partir de numerosos autores de este siglo).

La subfamilia Thelaxinae tiene un corto número de especies y la presencia de la subfamilia Hormaphidinae es casi testimonial en la región Paleártica (Dixon op. cit.). Consecuentemente con ello, en la zona estudiada hay pocas especies de estas dos subfamilias: tres de la primera (los tres únicos telaxinos conocidos en la Península Ibérica) y una de la segunda (la única que se desarrolla sobre plantas silvestres en el occidente europeo).

La representación de los Anoeciinae es también muy pobre (el 0,38\% del total de especies), pero entra dentro de lo esperable, pues en la Península Ibérica se han citado con certeza solamente tres especies.

La subfamilia Pemphiginae no está bien representada en la zona, 11 especies son pocas ciertamente (el $4,14 \%$, mientras que en la fauna mundial son el 7\% [Dixon, 1985] y en las de las montañas del oriente de Andalucía son el 8,1\% [Ghosh y Nieto Nafría, 1994]). La ausencia en el territorio de Pistacia spp., hospedoras primarias de las especies de Fordini, explica la pobreza de especies de esa tribu, de tal modo que Baizongia pistaciae, Forda formicaria y Paracletus spp., de las que se recogieron alados, tienen que mantenerse anholocíclicamente sobre sus respectivos hospedadores secundarios. La pérdida reciente, por la grafiosis, de los olmos (hospedadores primarios en este caso) puede explicar la pobreza de Eriosomatinae. La relativa penuria de Pemphiginae (cinco especies de Pemphigus, pues Thecabius affinis es la única de su género en la Península Ibérica) no puede tener una explicación similar, ya que los chopos son relativamente abundantes en las localidades de las clases altitudinales bajas, si bien es cierto que hoy en día no son frecuentes las choperas formadas por el autóctono Populus nigra. En cualquier caso, la ausencia de datos de algunas especies de esta subfamilia, así como la pobreza de los de otras, puede ser debida al régimen radicícola de los penfiginos en el hospedador secundario, que evidentemente dificulta su captura.

La representación de la subfamilia Drepanosiphinae es muy buena (30 especies, el $11,28 \%$ del total, porcentaje que se acerca al de la subfamilia en el Mundo, a partir de los datos de Dixon, op.cit., pues la separación de los Thelaxinae no tiene importancia porcentual). Esta buena representación puede deberse a la naturaleza euratlántica del territorio, que permite la presencia de hayas (Fagus sylvatica) y abedules (Betula celtiberica) y la diversidad de robles y encinas (Quercus spp.), sin olvidar la presencia de alisos, (Alnus glutinosa), nogales (Juglans regia), arces (Acer spp.), avellanos (Corylus avellana) y de algunos géneros de Leguminosae y Cyperaceae, que son hospedadoras de determinados géneros de pulgones.

También es relativamente alta la representación de la subfamilia Chaitophorinae (11 especies, el $4,14 \%$ del total), debido al elevado número de especies de Chaitophorus (6 especies de las 11 de este género citadas en España) que se desarrollan sobre árboles de los géneros Populus y Salix, que son muy abundantes en el territorio. Dixon (op.cit.) 
Tabla 1.- Relación de especies de pulgones recogidas en 92 localidades de la vertiente Sur de la Provincia fitogeográfica Orocantábrica, agrupadas por subfamilias y en Aphidinae por tribus. A la izquierda se señala la presencia de la especie en cada sector fitogeográfico (ver texto para la identificación de los sectores).- N: citas inéditas, B: citas bibliográficas, T: citas bibliográficas e inéditas. A la derecha se señala la presencia (X) de la especie en cada clase altitudinal I a VI (ver texto para la identificación de las clases altitudinales). Aún más a la derecha se señala la pertenencia de la especie a los grupos de inventarios, según se expone en el texto.- $\square$ : exclusiva del grupo, $\square$ : presencia en el grupo.

Tabla 1.- Relation of aphid species collected in 92 localities on the southern slope of the Orocantabrian phytogeographic Province, grouped in subfamilies and in Aphidinae by tribes. On the left the presence of the species in each phytogeographic sector is shown (see text for the identification of the sectors).- N: unpublished records, B: bibliographic records, T: bibliographic and unpublished records. On the right the presence $(\mathrm{X})$ for the species in each altitudinal level I to VI is shown (see text for identification of the altitudinal levels). Further to the right the species belonging to the plot groups is shown, from what is staded in the text.- $\square$ : exclusive to the group, $\square$ : presence in the group.

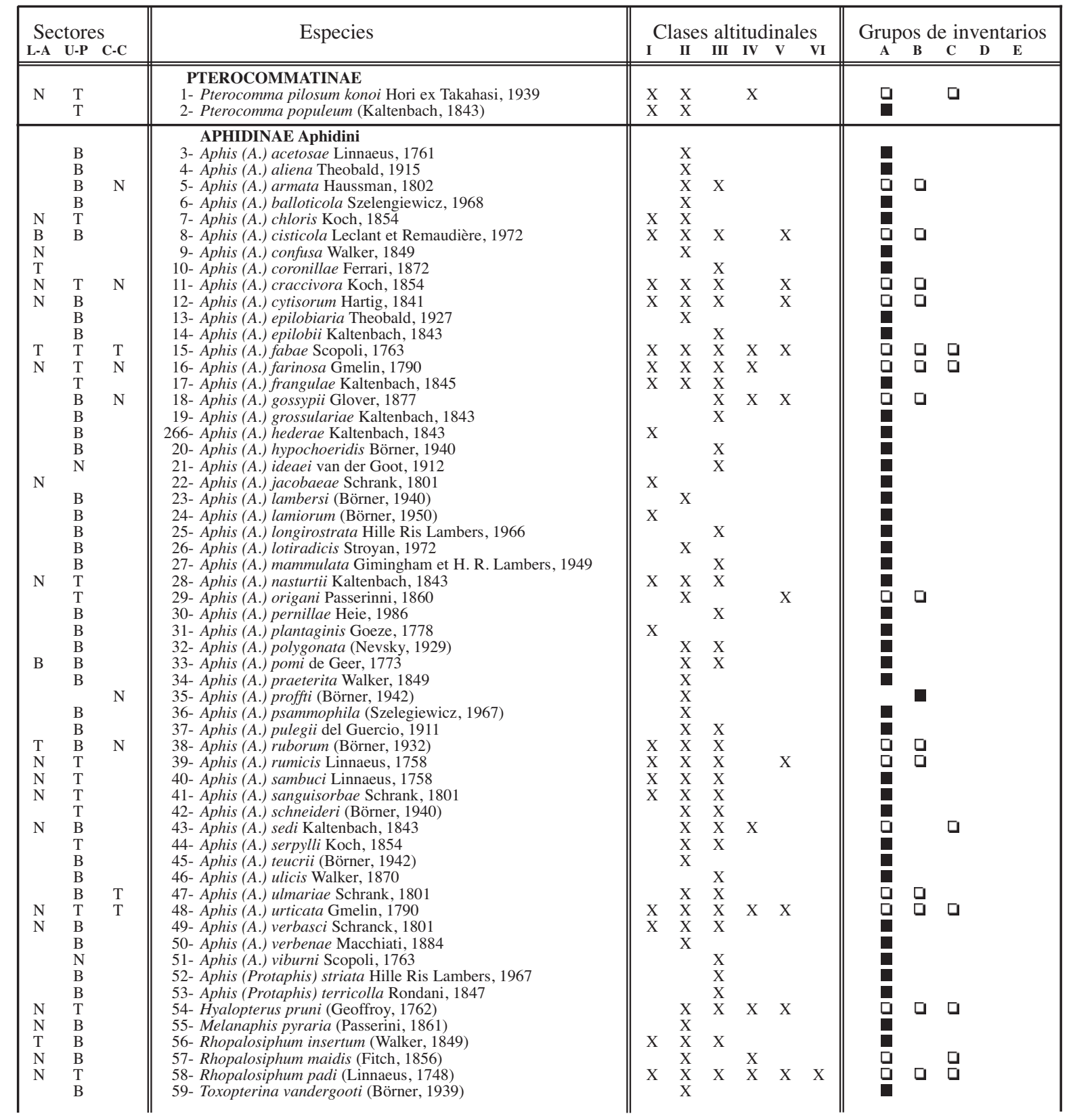


Tabla 1.- Cont.

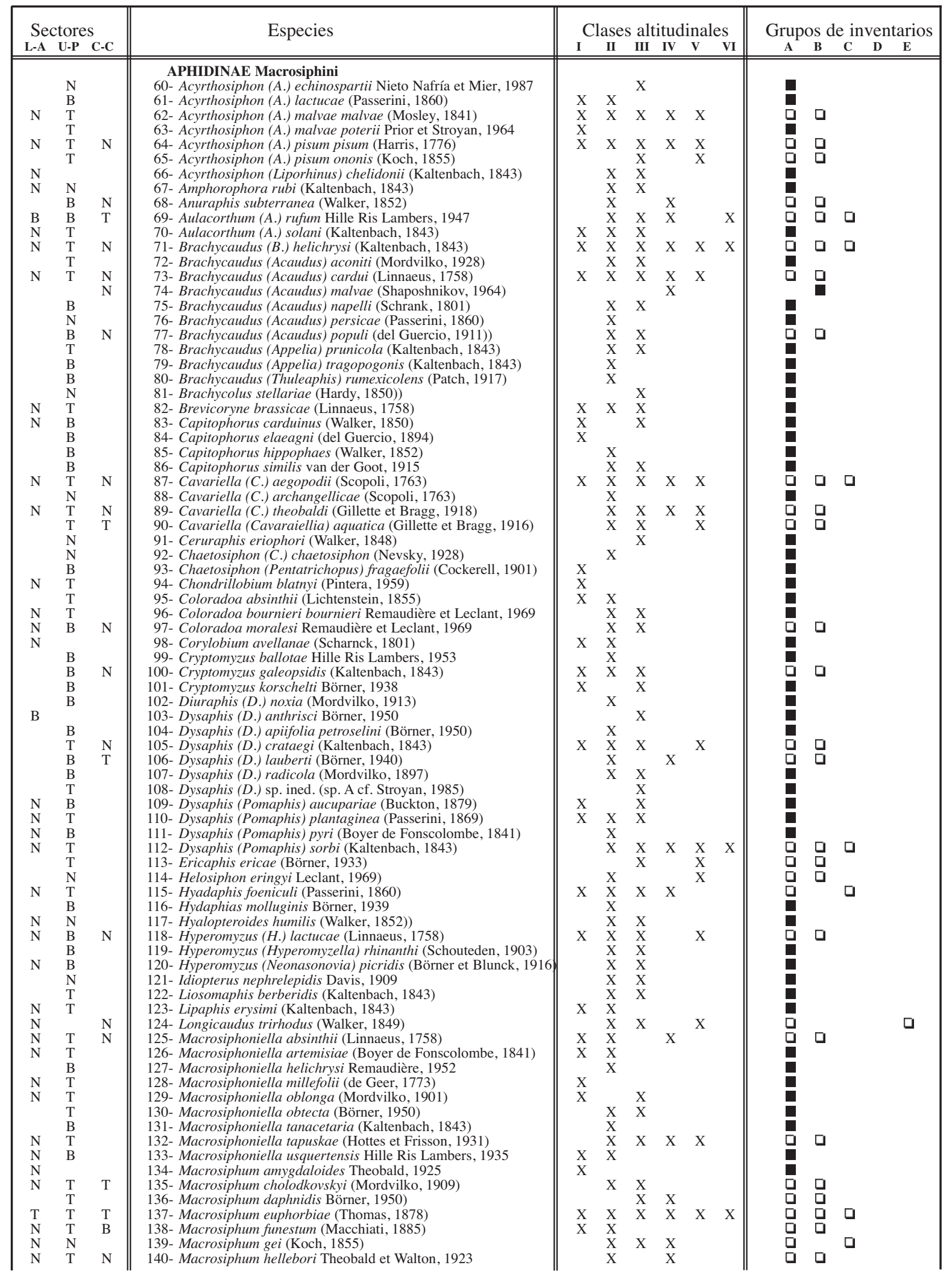


Tabla 1.- Cont.

\begin{tabular}{|c|c|c|c|c|c|c|c|c|c|c|c|c|c|}
\hline \multicolumn{3}{|c|}{ Sectores } & \multirow[t]{2}{*}{ Especies } & \multicolumn{6}{|c|}{ Clases altitudinales } & \multicolumn{4}{|c|}{ Grupos de inventarios } \\
\hline L-A & U-P & C-C & & I & II & III & IV & V & VI & A & B & C D & $\mathbf{E}$ \\
\hline $\mathrm{B}$ & $\bar{B}$ & $\overline{\mathrm{B}}$ & 141- Macrosiphum meixneri Börner, 1950 & & $\bar{X}$ & $\overline{\mathrm{X}}$ & $\overline{\mathrm{X}}$ & & $\mathrm{X}$ & $\square$ & $\square$ & $\begin{array}{ll}\square & \square\end{array}$ & \\
\hline $\mathrm{N}$ & $\mathrm{T}$ & $\mathrm{N}$ & 142- Macrosiphum rosae (Linnaeus, 1758) & $\mathrm{X}$ & $\mathrm{X}$ & $\mathrm{X}$ & $\mathrm{X}$ & $\mathrm{X}$ & & $\bar{\square}$ & $\bar{\square}$ & $\overline{\bar{u}}$ & ב \\
\hline $\mathrm{N}$ & $\mathrm{T}$ & & 143- Macrosiphum stellariae Theobald, 1913 & & $\mathrm{X}$ & $\mathrm{X}$ & $\mathrm{X}$ & & & $\square$ & $\bar{\square}$ & $\bar{\square}$ & \\
\hline $\mathrm{N}$ & $\mathrm{N}$ & & 144- Megoura viciae Buckton, 1876 & & $\mathrm{X}$ & $\mathrm{X}$ & & & & $\mathbf{E}$ & & & \\
\hline & $\mathrm{T}$ & & 145- Metopeuron fuscoviride Stroyan, 1950 & $\mathrm{X}$ & $\mathrm{X}$ & $\mathrm{X}$ & & & & $=$ & & & \\
\hline & $\mathrm{N}$ & & 146- Metopolophium dirhodum (Walker, 1849) & & & & $\mathrm{X}$ & & & & $\mathbf{a}$ & & \\
\hline & $\mathrm{N}$ & $\mathrm{N}$ & 147- Metopolophium festucae festucae (Theobald, 1917) & & $\mathrm{X}$ & & $\mathrm{X}$ & $\mathrm{X}$ & & & & & \\
\hline $\mathrm{N}$ & $\mathrm{N}$ & $\mathrm{N}$ & 148- Metopolophium festucae cerealium Stroyan, 1982 & $\mathrm{X}$ & & $\mathrm{X}$ & $\mathrm{X}$ & $\mathrm{X}$ & & $\square$ & $\bar{\square}$ & & \\
\hline $\mathrm{N}$ & $\mathrm{T}$ & $\mathrm{N}$ & 149- Metopolophium montanum Hille Ris Lambers, 1966 & & $\mathrm{X}$ & & $\mathrm{X}$ & $\mathrm{X}$ & $\mathrm{X}$ & $\bar{\square}$ & $\bar{\square}$ & ב & ב \\
\hline $\mathrm{N}$ & & & 150- Microlophium carnosum (Buckton, 1876) & $\mathrm{X}$ & $\mathrm{X}$ & & & & & $\overline{\mathbf{u}}$ & & & \\
\hline & B & & 151- Microsiphum millefolii Wahlgren, 1940 & $\mathrm{X}$ & & & & & & & & & \\
\hline $\mathrm{N}$ & $\mathrm{T}$ & $\mathrm{N}$ & 152- Myzaphis rosarum (Kaltenbach, 1843) & $\mathrm{X}$ & $\mathrm{X}$ & $\mathrm{X}$ & & $\mathrm{X}$ & & $\bar{\square}$ & & & $\square$ \\
\hline $\mathrm{N}$ & $\mathrm{N}$ & $\mathrm{N}$ & 153-Myzus (M.) cerasi (Fabricius, 1775) & & $\mathrm{X}$ & $\mathrm{X}$ & $\mathrm{X}$ & & $\mathrm{X}$ & 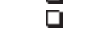 & $\square$ & $\square$ & \\
\hline & $\mathrm{T}$ & & 154- Myzus (M.) lytrhi (Schrank, 1801) & & $\mathrm{X}$ & $\mathrm{X}$ & & & & 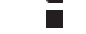 & & & \\
\hline $\mathrm{N}$ & $\mathrm{B}$ & & 155- Myzus (M.) ornatus Laing, 1932 & & $\mathrm{X}$ & $\mathrm{X}$ & & & & $=$ & & & \\
\hline $\mathrm{N}$ & $\mathrm{T}$ & $\mathrm{N}$ & 156- Myzus (Nectarosiphon) ascalonicus Doncaster, 1946 & & $\mathrm{X}$ & $\mathrm{X}$ & $\mathrm{X}$ & & & $\bar{\square}$ & 무 & $\square$ & \\
\hline & $B$ & B & 157- Myzus (Nectarosiphon) ligustri (Mosley, 1841) & & $\mathrm{X}$ & & & & & 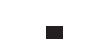 & $\mathbf{\square}$ & & \\
\hline $\mathrm{N}$ & $\mathrm{T}$ & & & $\mathrm{Y}$ & $\mathrm{X}$ & & & & & 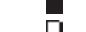 & $\square$ & & \\
\hline $\mathrm{N}$ & & & $\begin{array}{l}\text { 159- Myzus (Nectarosiphon) persicae (Sulzer, 1776) } \\
\text { 160- Myzus (Scyamyzus) cymbalariae Stroyan, 1954) }\end{array}$ & $X$ & $\mathrm{X}$ & $\begin{array}{l}\mathrm{X} \\
\mathrm{X}\end{array}$ & $\begin{array}{l}\mathrm{X} \\
\mathrm{X}\end{array}$ & $\mathrm{X}$ & & 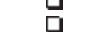 & $\square$ & $\square$ & \\
\hline $\mathrm{N}$ & B & & 161- Nasonovia (N.) nigra (Hille Ris Lambers, 1931) & & $\mathrm{X}$ & $\mathrm{A}$ & $\mathrm{X}$ & & & 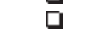 & & $\square$ & \\
\hline & $\mathrm{N}$ & & 162- Nasonovia (N.) pilosellae (Börner, 1933) & & & $\mathrm{X}$ & & & & & & & \\
\hline $\mathrm{N}$ & $\mathrm{T}$ & & 163- Nasonovia (N.) ribisnigri (Mosley, 1841) & $\mathrm{X}$ & $\mathrm{X}$ & $\mathrm{X}$ & & & & & & & \\
\hline $\mathrm{N}$ & $\mathrm{T}$ & $\mathrm{N}$ & 164- Nasonovia (Neokakimia) sp & & $\mathrm{X}$ & $\mathrm{X}$ & $\mathrm{X}$ & $\mathrm{X}$ & & $\bar{\square}$ & 口 & ב & \\
\hline $\mathrm{N}$ & $\mathrm{T}$ & $\mathrm{N}$ & 165- Nearctaphis bakeri (Cowen, 1895) & $\mathrm{X}$ & $\mathrm{X}$ & $\mathrm{X}$ & $\mathrm{X}$ & & & $\bar{\square}$ & $\bar{\square}$ & $\bar{\square}$ & \\
\hline & B & & 166- Ovatomyzus chamaedrys (Passerini, 1879) & $\mathrm{X}$ & & & & & & $\overline{\mathbf{u}}$ & & & \\
\hline & B & $\mathrm{N}$ & 167- Ovatus crataegarius (Walker, 1850) & $\mathrm{X}$ & $\mathrm{X}$ & $\mathrm{X}$ & $\mathrm{X}$ & $\mathrm{X}$ & & $\bar{\square}$ & $\square$ & & \\
\hline & $\mathrm{B}$ & & 168- Paramyzus heraclei Börner, 1933 & $\mathrm{X}$ & $\mathrm{X}$ & & & & & $\overline{\mathbf{z}}$ & & & \\
\hline $\mathrm{N}$ & $\mathrm{T}$ & $\mathrm{N}$ & 169- Phorodon humuli (Schrank, 1901) & $\mathrm{X}$ & $\mathrm{X}$ & $\mathrm{X}$ & & & & $\bar{\square}$ & ב & & \\
\hline $\mathrm{N}$ & $\mathrm{B}$ & & 170- Pleotrichophorus glandulosus (Kaltenbach, 1846) & $\mathrm{X}$ & & $\mathrm{X}$ & & & & & & & \\
\hline & $\mathrm{N}$ & & 171- Rhodobium porosum (Sanderson, 1900)) & & & & & $\mathrm{X}$ & & & 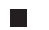 & & \\
\hline $\mathrm{N}$ & & & 172- Rhopalosiphoninus (R.) latysiphon (Davidson, 1912) & & & $\mathrm{X}$ & & & & & & & \\
\hline & $\mathrm{T}$ & & 173- Roepkea marchali (Börner, 1931) & & $\mathrm{X}$ & $\mathrm{X}$ & & & & & & & \\
\hline $\mathrm{N}$ & B & & 174- Semiaphis dauci (Fabricius, 1775) & & $\mathrm{X}$ & $\mathrm{X}$ & & & & E & & & \\
\hline $\mathrm{T}$ & $\mathrm{T}$ & $\mathrm{T}$ & 175- Sitobion avenae (Fabricius, 1775) & $\mathrm{X}$ & $\mathrm{X}$ & $\mathrm{X}$ & $\mathrm{X}$ & $\mathrm{X}$ & $\mathrm{X}$ & 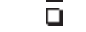 & 口 & 口 & \\
\hline $\mathrm{T}$ & $\mathrm{T}$ & $\mathrm{T}$ & 176- Sitobion fragariae (Walker, 1848) & $\mathrm{X}$ & $\mathrm{X}$ & $\mathrm{X}$ & $\mathrm{X}$ & $\mathrm{X}$ & $\mathrm{X}$ & $\bar{\square}$ & $\bar{\square}$ & $\bar{\square}$ & \\
\hline $\mathrm{N}$ & $\mathrm{N}$ & $\mathrm{N}$ & 177- Staegeriella necopinata (Börner, 1939) & & $\mathrm{X}$ & $\mathrm{X}$ & & & & $=$ & $\bar{\square}$ & & \\
\hline $\mathrm{T}$ & & & 178- Uroleucon (U.) achilleae (Koch, 1855) & & $\mathrm{X}$ & $\mathrm{X}$ & & & & 口 & & & \\
\hline $\mathrm{B}$ & $\mathrm{T}$ & & 179- Uroleucon (U.) cichorii cichorii (Koch, 1855) & $\mathrm{X}$ & $\mathrm{X}$ & $\mathrm{X}$ & $\mathrm{X}$ & & $\mathrm{X}$ & ב & & $\square$ & \\
\hline & B & & 180- Uroleucon (U.) chichorii grosum (Hille Ris Lambers, 1939) & & $\mathrm{X}$ & & & & & & & & \\
\hline $\mathrm{N}$ & $\mathrm{T}$ & & 181- Uroleucon (U.) chondrillae (Nevsky, 1929) & $\mathrm{X}$ & & $\mathrm{X}$ & & & & E & & & \\
\hline $\mathrm{N}$ & B & & 182- Uroleucon (U.) hypochoeridis (Fabricius, 1779) & $\mathrm{X}$ & $\mathrm{X}$ & $\mathrm{X}$ & & & & & & & \\
\hline & $\mathrm{T}$ & & 183-Uroleucon (U.) picridis (Fabricius, 1775) & & $\mathrm{X}$ & & & & & $=$ & & & \\
\hline $\mathrm{N}$ & B & $\mathrm{N}$ & 184- Uroleucon (U.) Sonchi (Linnaeus, 1767) & $\mathrm{X}$ & $\mathrm{X}$ & $\mathrm{X}$ & & & & [ & $\square$ & & \\
\hline & $\mathrm{B}$ & & 185- Uroleucon (U.) tussilaginis (Walker, 1850) & & $\mathrm{X}$ & & & & & $\overline{\mathbf{n}}$ & & & \\
\hline $\mathrm{N}$ & $\mathrm{B}$ & $\mathrm{N}$ & 186- Uroleucon (U.) sp. ined., aff. picridis & $\mathrm{X}$ & $\mathrm{X}$ & $\mathrm{X}$ & & & & $\bar{\square}$ & $\square$ & & \\
\hline $\mathrm{N}$ & & & 187- Uroleucon (Lambersius) erigeronensis (Thomas, 1878) & $\mathrm{X}$ & & & & & & & & & \\
\hline $\mathrm{N}$ & $\mathrm{N}$ & & 188- Uroleucon (Uromelan) aeneum (Hille Ris Lambers, 1939) & $\mathrm{X}$ & $\mathrm{X}$ & $\mathrm{X}$ & & & & & & & \\
\hline $\mathrm{N}$ & $\mathrm{B}$ & & 189- Uroleucon (Uromelan) campanulae (Kaltenbach, 1843) & $\mathrm{X}$ & & & $\mathrm{X}$ & & & $\bar{\square}$ & $\square$ & & \\
\hline $\mathrm{N}$ & $\mathrm{T}$ & & 190- Uroleucon (Uromelan) jaceae (Linnaeus, 1758) & $\mathrm{X}$ & $\mathrm{X}$ & $\mathrm{X}$ & & & & $=$ & & & \\
\hline & B & & 191- Uroleucon (Uromelan) simile (Hille Ris Lambers, 1935) & & & $\mathrm{X}$ & & & & & & & \\
\hline $\mathrm{N}$ & & & 192- Uroleucon (Uromelan) solidaginis (Fabricius, 1779) & $\mathrm{X}$ & $\mathrm{X}$ & & $\mathrm{X}$ & & $\mathrm{X}$ & $\square$ & & $\square$ & \\
\hline & $\mathrm{T}$ & & 193- Wahlgreniella arbuti (Davidson, 1920) & $\mathrm{X}$ & & & & & & & & & \\
\hline & $\mathrm{N}$ & & 194- Wahlgreniella nervata (Gillette, 1908) & $\mathrm{X}$ & & & & & & ( & & & \\
\hline & $\mathrm{T}$ & & 195- Wahlgreniella ossiannilssoni Hille Ris Lambers, 1949 & $\mathrm{X}$ & & & & & & U & & & \\
\hline & $\mathrm{N}$ & & 196- Baizongia pistaciae (Linnaeus, 1767) & & $\mathrm{X}$ & & & & & & & & \\
\hline & $\mathrm{N}$ & & 197- Eriosoma (Schizoneura) lanuginosum (Hartig, 1841)) & & $\mathrm{X}$ & & & & & $\mathbf{\square}$ & & & \\
\hline & $\mathrm{T}$ & & 198- Eriosoma (Schizoneura) ulmi (Linnaeus, 1758) & & $\mathrm{X}$ & & & & & $=$ & & & \\
\hline & $\mathrm{N}$ & & 199- Forda formicaria von Heyden, 1837 & & $\mathrm{X}$ & & & & & $=$ & & & \\
\hline & $\mathrm{N}$ & & 200- Paracletus cimiciformis von Heyden, 1837 & & & & & $\mathrm{X}$ & & & $\mathbf{\square}$ & & \\
\hline & $\mathrm{N}$ & & 201- Paracletus donisthorpei Theobald, 1927 & & $\mathrm{X}$ & & & & & & & & \\
\hline & $\mathrm{T}$ & & 202- Pemphigus bursarius (Linnaeus, 1758) & $\mathrm{X}$ & $\mathrm{X}$ & & & & & & & & \\
\hline $\mathrm{N}$ & B & & 203-Pemphigus populi Courchet, 1879 & & $\mathrm{X}$ & $\mathrm{X}$ & & & & $\bar{\square}$ & $\square$ & & \\
\hline & $\mathrm{T}$ & & 204- Pemphigus populinigrae (Schrank, 1801) & & $\mathrm{X}$ & $\mathrm{X}$ & & & & $\overline{\mathbf{Z}}$ & & & \\
\hline $\mathrm{N}$ & $\mathrm{T}$ & & 205- Pemphigus spirothecae Passerini, 1856 & $\mathrm{X}$ & $\mathrm{X}$ & $\mathrm{X}$ & & & & $\overline{\mathbf{a}}$ & & & \\
\hline $\mathrm{N}$ & $\mathrm{T}$ & & 206- Thecabius affinis (Kaltenbach, 1843) & & $\mathrm{X}$ & $\mathrm{X}$ & $\mathrm{X}$ & & & $\bar{\square}$ & & $\square$ & \\
\hline & $\mathrm{T}$ & & $\begin{array}{c}\text { ANOECIINAE } \\
\text { 207- Anoecia corni (Fabricius, 1775) }\end{array}$ & & $\mathrm{X}$ & & & & & ] & & & \\
\hline & B & & $\begin{array}{l}\text { HORMAPHIDINAE } \\
\text { 208- Hammamelistes betulinus (Horvath, 1896) }\end{array}$ & & $\mathrm{X}$ & & & & & [ & & & \\
\hline & $\mathrm{N}$ & & $\begin{array}{l}\text { THELAXINAE } \\
\text { 209- Glyphina betulae (Linnaeus, 1758) }\end{array}$ & & & $\mathrm{X}$ & & & & 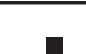 & & & \\
\hline $\mathrm{N}$ & T & & 210- Thelaxes dryophila (Schrank, 1801) & & $\mathrm{X}$ & $\lambda$ & $\mathrm{X}$ & $\mathrm{X}$ & & $\square$ & $\square$ & $\square$ & \\
\hline $\mathrm{N}$ & & & 211- Thelaxes suberi (Del Guercio, 1911) & & & & $\mathrm{X}$ & & & & & & \\
\hline $\mathrm{T}$ & $\mathrm{T}$ & & $\begin{array}{l}\text { DREPANOSIPHINAE } \\
\text { 212- Appendiseta robiniae (Gillette, 1907) }\end{array}$ & $\mathrm{X}$ & $\mathrm{X}$ & & & & & D & & & \\
\hline & $\mathrm{T}$ & & 213- Betulaphis quadrituberculata (Kaltenbach, 1843) & $\begin{array}{l}\lambda \\
X\end{array}$ & & $\mathrm{X}$ & & & & 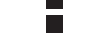 & & & \\
\hline $\mathrm{N}$ & & & 214- Calaphis betulicola (Kaltenbach, 1843) & & & & $\mathrm{X}$ & & & & & Q & \\
\hline $\mathrm{N}$ & $\mathrm{T}$ & B & 215- Calaphis flava (Mordvilko, 1928 ) & $\mathrm{X}$ & $\mathrm{X}$ & $\mathrm{X}$ & $\mathrm{X}$ & & & $\square$ & $\square$ & & \\
\hline
\end{tabular}


Tabla 1.- Cont.

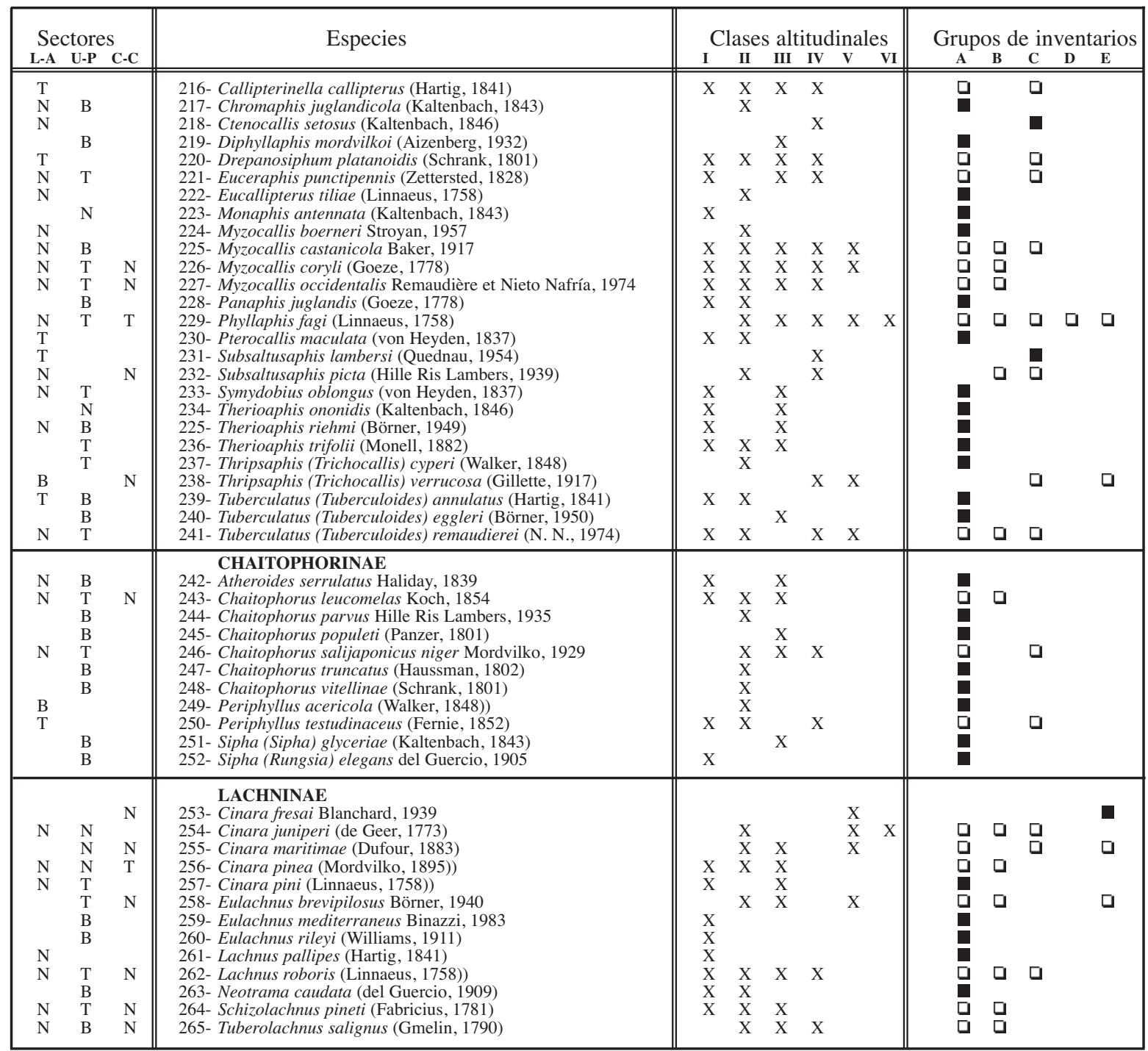

fija un $4 \%$ para esta subfamilia en la afidofauna mundial. En ésta y en la anterior subfamilia la representación porcentual es similar a la registrada en las montañas orientales de Andalucía (Ghosh y Nieto Nafría, op. cit.).

La subfamilia Lachninae representa, con 13 especies, el 4,89\% del total de especies de la zona, cifra que está muy por debajo del $9 \%$ mundial y del $10,8 \%$ de las mencionadas montañas andaluzas. El responsable de esa pobreza es el bajo número de especies del género Cinara (con mucho el más importante de la subfamilia), que puede estar en relación con la parca representación en el área de sus hospedadores, cupresáceas.

Evidentemente, la subfamilia Aphidinae (sensu Dixon, 1985) es la más representada: sus 196 especies constituyen el 73,68\% de las especies de pulgones presentes en el territorio. De ellas: 2 son consideradas aquí Pterocommatinae y las 194 restantes Aphidinae, sensu stricto, de ellas 58 Aphidini (con claro predominio del género Aphis, 52 especies) y 136 son Macrosiphini. Estas dos subfamilias, como las entendemos aquí, representan en la fauna mundial un $60 \%$ (Dixon, op. cit.). La diferencia entre 
esos dos porcentajes se explica por la relativa pobreza, ya comentada, de Pemphiginae y Lachninae, además de atenuarse porque el conjunto Greenideinae (sin representación paleárticooccidental) más Hormaphidinae tiene el $7 \%$ de la fauna mundial (Dixon, op. cit.).

\section{DISTRIBUCIÓN POR SECTORES}

El sector UP es el más rico, con 234 especies, seguido a distancia por el sector LA con 137; y el sector CC es el más pobre según nuestros datos, con 62 especies. El conocimiento de la afidofauna de cada uno de los sectores obtenido a partir de las citas bibliográficas y de las inéditas es también muy diferente (tabla 2); así se citan por vez primera 34 especies en el sector UP (el de mejor conocimiento previo, por citas bibliográficas), 46 en el sector CC y 114 en el sector LA.

Por otra parte (tabla 2), el sector UP tiene más especies exclusivas (112, el 42,10\% del total)) que cualquiera de los otros dos, y hay notablemente más especies exclusivas en el sector LA que en el sector CC.

La mayor riqueza y diversidad observada en el sector UP ha de tener base real, habida cuenta de las características florísticas (Rivas Martínez et al.op. cit.), aunque las diferencias entre el sector UP y los otros dos han de estar algo acentuadas por la mayor atención que se le ha dedicado (especialmente por Tizado Morales, 1991) como se aprecia al comparar las especies que en los tres sectores se conocen solamente por citas inéditas $(\mathrm{N})$ con las conocidas por citas inéditas y bibliográficas $(\mathrm{N}+\mathrm{T})$, ver tabla 2.

\section{DisTRIBUCIÓN POR CLASES ALTITUDINALES}

De la clase I (800 a 999) citamos 114 especies, de la II (1.000 a 1.199) 190 especies, de la III (1.200 a 1.399) 154 especies, el número de especies baja a 68 en la clase IV (1.400 a 1.599$)$ y a 48 especies en la V (1.600 a 1.799), por último solamente hemos registrado 14 especies en la clase VI (1.800 a 1.999). Como se puede apreciar los niveles II y III son los de mayor riqueza, en relación con una relativa mayor riqueza florística; por ello examinamos
Tabla 2.- Número de especies citadas en cada sector fitogeográfico (UP, LA y CC), en total y a partir de citas bibliográficas solamente (B), de citas bibliográficas e inéditas (T) y de citas inéditas solamente $(\mathrm{N})$, señalándose las especies de las que se dan nuevas citas $(\mathrm{N}+\mathrm{T})$ y las especies exclusivas de cada sector según los datos que se aportan.

Tabla 2.- Number of species reported in each phytogeographic sector (UP, LA and CC), in total and only from bibliographic records (B), from bibliographic and unpublished records $(\mathrm{T})$ and only from unpublished records $(\mathrm{N})$, showing the species which are given new citations $(\mathrm{N}+\mathrm{T})$ and the species exclusive to each sector from the data given.

\begin{tabular}{|c|c|c|c|c|c|c|}
\hline \multirow{2}{*}{ Sector } & \multirow{2}{*}{$\begin{array}{l}\text { especies } \\
\text { en total }\end{array}$} & \multicolumn{4}{|c|}{ especies con citas } & \multirow{2}{*}{$\begin{array}{l}\text { especies } \\
\text { exclusivas }\end{array}$} \\
\hline & & $\mathrm{B}$ & $\mathrm{T}$ & $\mathrm{N}$ & $\mathrm{N}+\mathrm{T}$ & \\
\hline UP & 234 & 103 & 97 & 34 & 131 & 112 \\
\hline LA & 137 & 8 & 15 & 114 & 129 & 25 \\
\hline $\mathrm{CC}$ & 62 & 4 & 12 & 46 & 58 & 3 \\
\hline
\end{tabular}

a continuación la situación en el nivel más bajo (I) y en los niveles altos ( $\mathrm{V} \mathrm{y} \mathrm{VI).}$

En la clase altitudinal I se nota la ausencia de algunas especies presentes en las clases inmediatas (II, III). Desde una visión meramente "naturalista" y de conocedores de los pulgones, este hecho es perfectamente explicable, bien por la ubicación en ese territorio de sus respectivas (y obligadas) plantas hospedadoras (que no descienden tanto) o por la atlanticidad de las especies afidianas, estimando la atlanticidad en función de la existencia o mayor frecuencia de citas en zonas europeo-atlánticas y ausencia o menor frecuencia de ellas en zonas mediterráneas. Así, por ejemplo, puede suceder con las especies que se relacionan en la tabla 3.

La ausencia en esa clase altitudinal de otras especies (Thelaxes suberi, Chromaphis juglandicola, Ctenocallis setosus, Diphyllaphis mordvilkoi, las especies de Chaitophorus no incluidas en la tabla 3, Cinara spp., Tuberolachnus salignus, muchas especies del género Aphis [que por brevedad no se relacionan], Hyalopterus pruni, Rhopalosiphum maidis, varias especies de Acyrthosiphon, las especies de Brachycaudus y de Dysaphis no incluidas en la tabla 3, Capitophorus spp., Coloradoa spp., Helosiphon eringyi, Hydaphias molluginis, Hyalopteroides humilis, Liosomaphis berberidis, 
Tabla 3.- Especies de pulgones, con las plantas sobre las que se recogieron, no presentes en la clase altitudinal I y presentes en las clases inmediatas superiores del Sur de la Orocantábrica, denotadoras de atlanticidad.

Tabla 3.- Aphid species (with the plants they were collected on) not present in altitudinal level I and present in immediate upper levels in the south of the Orocantabrian, which indicate oceanic characteristics.

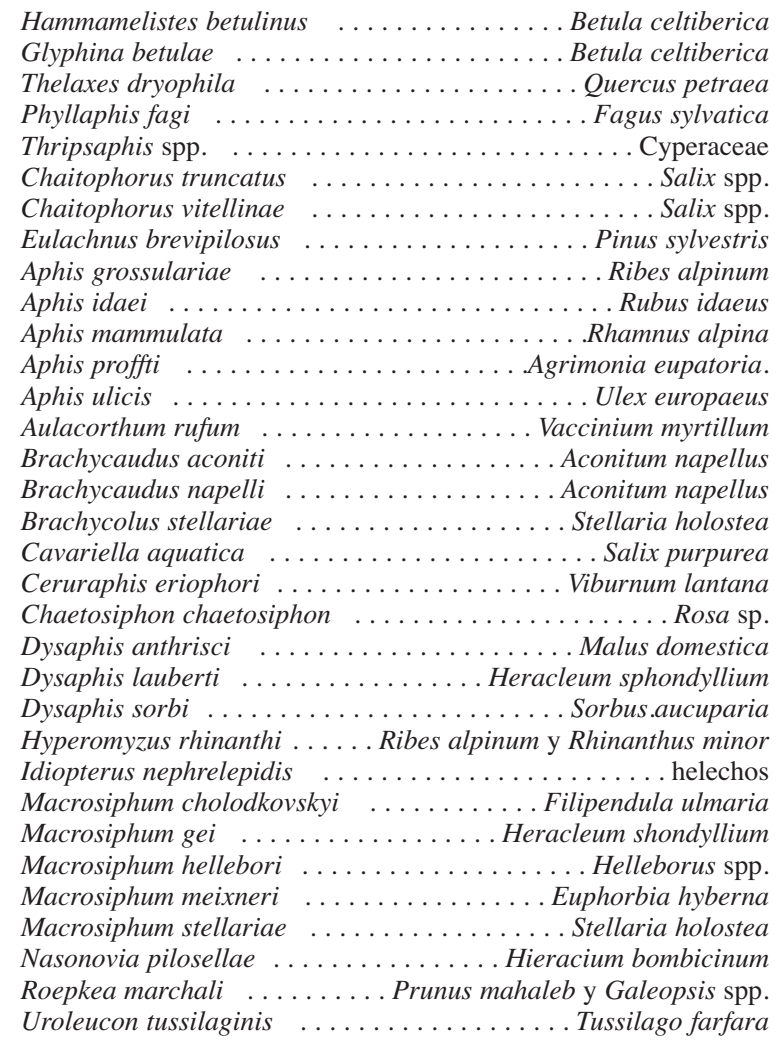

Megoura viciae, Rhodobium porosum, las especies de Macrosiphoniella, de Macrosiphum, de Metopolophium, de Myzus, de Nasonovia y de Uroleucon y las de la subfamilia Pemphiginae) solamente se puede explicar porque sean poco frecuentes en la zona. Es decir, el no haberlas capturado puede deberse a su rareza y no a su ausencia absoluta.

Actuando desde similares principios, con las especies que alcanzan los niveles más elevados (V o VI) podemos establecer dos grupos, sin considerar Cinara fresai, cuya identificación es dudosa. Un grupo está constituido por especies de amplia dis- tribución general o ibérica (a partir de la bibliografía disponible o de los datos de nuestra [N.N. y M.D.] colección) y el otro grupo por las pocas especies que son marcadamante orófilas en la Península Ibérica, ya que en la Europa nordpirenaica algunas no lo son (interviene aquí el conocido efecto altitud-latitud). Las especies del primer grupo son muy numerosas y no las relacionamos, evidentemente son aquéllas con presencia en las clases V y VI (ver fig. 1) y no pertenecientes al segundo grupo. Éste está formado por Thripsaphis verrucosa (de ciperáceas, en zonas frescas), Eulachnus brevipilosus (de Pinus sylvestris y especies próximas), Aulacorthum rufum (enfeudada en Vaccinum), Cavariella aquatica (cuyo único hospedador primario es Salix purpurea), Dysaphis sorbi (cuyo hospedador primario es el serbal de cazadores), Macrosiphum meixneri (limitada aquí a Euphorbia hyberna) y Nasonovia sp. del subgénero Neokakimia (monoica sobre Saxifraga). La relación de especies orófilas en el sentido expresado se puede incrementar con algunas otras que no llegan a las altitudes de las clases V y VI, pero que se encuentran en localidades de la IV, es el caso de Dysaphis lauberti (cuyo hospedador primario es el manzano), Macrosiphum daphnidis (que vive sobre Daphne laureola), $M$. stellariae (limitado a Stellaria holostea) y Nasonovia nigra (que vive sobre varias compuestas ligulifloras), de los drepanosifinos propios de abedules y de las especies de Subsaltusaphis (monoicas sobre diversas ciperáceas). Todas estas especies no están necesariamente restringidas a los niveles más altos, pues pueden, en mayor o menor medida, presentarse en niveles más bajos si se encuentran allí sus correspondientes plantas nutricias.

\section{DISTRIBUCIÓN CONJUNTA POR SECTORES Y CLASES ALTITUDINALES}

Tras ensayar un análisis en el que se consideraron por separado las 92 localidades y las especies capturadas en ellas, que no dió resultados, por la heterogeneidad de la información, desarrollamos el análisis expuesto en el apartado de material y métodos. El análisis de similitud entre inventarios nos permite considerar la distribución conjunta de las especies de pulgones en los sectores fitogeográ- 
ficos y en las clases altitudinales de la Provincia fitogeográfica Orocantábrica; además puede corroborar o rechazar las consideraciones acabadas de exponer. Como resultado del análisis de agrupamiento de los 15 inventarios configurados (fig. 2) se obtuvo un dendrograma (fig. 3), en el que fácilmente se delimitan cinco grupos (fig. 4), dos de los cuales están integrados por un solo inventario. En las columnas de la derecha de la tabla 1 se señalan la presencia y en su caso exlusividad de las especies en los inventarios. Como se puede apreciar 93 especies se encuentran en dos o más grupos (tabla 1).

El grupo A se halla constituido por los seis inventarios de los sectores UP y LA inferiores a

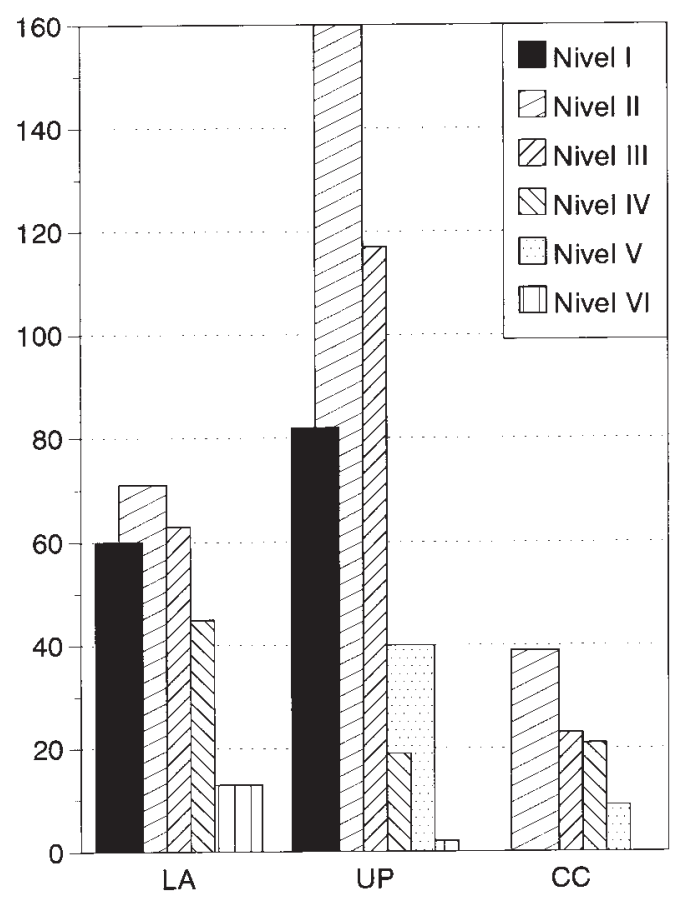

Fig. 2.- Número de especies por sector fitogeográfico y clase altitudinal; CC: Campurriano-Carrionés, UP: UbiñensePicoeuropeano, y LA: Laciano-Ancarense; Clases altitudinales: I, menos de 999 m, II: entre 1.000 y $1.199 \mathrm{~m}$, III, entre 1.200 y $1.399 \mathrm{~m}, \mathrm{IV}$, entre 1.400 y $1.599 \mathrm{~m}, \mathrm{~V}$, entre 1.600 y $1.799 \mathrm{~m}, \mathrm{y} \mathrm{VI}$, entre 1.800 y $1.999 \mathrm{~m}$.

Fig. 2.- Number of species per phytogeographic sector and altitudinal level; CC: Campurriano-Carrionés, UP: UbiñensePicoeuropeano, and LA: Laciano-Ancarense; altitudinal levels: I, less than 999 m, II: between 1,000 and 1,199 m, III, between 1,200 and $1,399 \mathrm{~m}, \mathrm{IV}$, between 1,400 and $1,599 \mathrm{~m}, \mathrm{~V}$, between 1,600 and $1,799 \mathrm{~m}$, and VI, between 1,800 and $1,999 \mathrm{~m}$.
1.399 m, es decir UP-I, UP-II, UP-III, LA-I, LA-II y LA-III. El grupo cuenta con un total de 252 especies, de las cuales 161 son exclusivas del mismo.

El grupo $\mathbf{B}$ está formado por cinco inventarios: UP-IV y UP-V (entre 1.400 y $1.799 \mathrm{~m}$ ) y CC-II, CC-III y CC-IV (entre 1.000 y $1.599 \mathrm{~m}$ ). El grupo cuenta con 81 especies de las cuales siete, solamente, son exclusivas del mismo.

El grupo $\mathbf{C}$ se halla formado por los inventarios del sector LA comprendidos entre los $1.400 \mathrm{y}$ los $1.999 \mathrm{~m}$ y cuenta con 48 especies de áfidos, cuatro de ellas exclusivas del grupo.

Los grupos D y $\mathbf{E}$ son los de inventario único. El inventario UP-VI (entre 1.600 y $1.799 \mathrm{~m}$ ), en el grupo D, presenta sólo dos especies, ninguna exclusiva. El inventario CC-V (1.600 a 1.799) es el único del grupo $\mathbf{E}$, con nueve especies, una de ellas exclusiva.

Efectivamente (ver "Distribución por sectores"), el sector UP es con mucho el más rico y el $\mathrm{CC}$ el más pobre en especies (fig. 2). La distribución general por clases altitudinales (ver el apartado correspondiente) se repite prácticamente en cada uno de los sectores (fig. 2), con menores diferencias en las cuatro clases inferiores (I a IV) del sector LA entre sí, que entre las mismas cuatro del sector UP. Como se aprecia con claridad el número de especies disminuye con la altura, como es esperable considerando la biología de las especies de pulgones y la composición florística de la zona.

De la agrupación existente, apreciable en el dendrograma (fig. 3), se puede concluir fácilmente la existencia de un gradiente altitudinal, aunque no sea equivalente en los tres sectores fitogeográficos, ya que están implicados diferentes grupos de inventarios (fig. 4).

Se aprecia además un gradiente que va de este a oeste y que afecta a los sectores UP y CC, igualando los inventarios medios-altos de UP con los medios-bajos de CC (grupo B). Una posible explicación a este hecho radica en la disminución de precipitaciones estivales hacia occidente y en la mayor continentalidad climática del sector CC (Rivas Martínez et al., 1984).

La composición del grupo $\mathbf{C}$ es relativamente heterogénea, pues podría esperarse que el inventario LA-IV se colocara cerca de los LA-I, II y III, y 


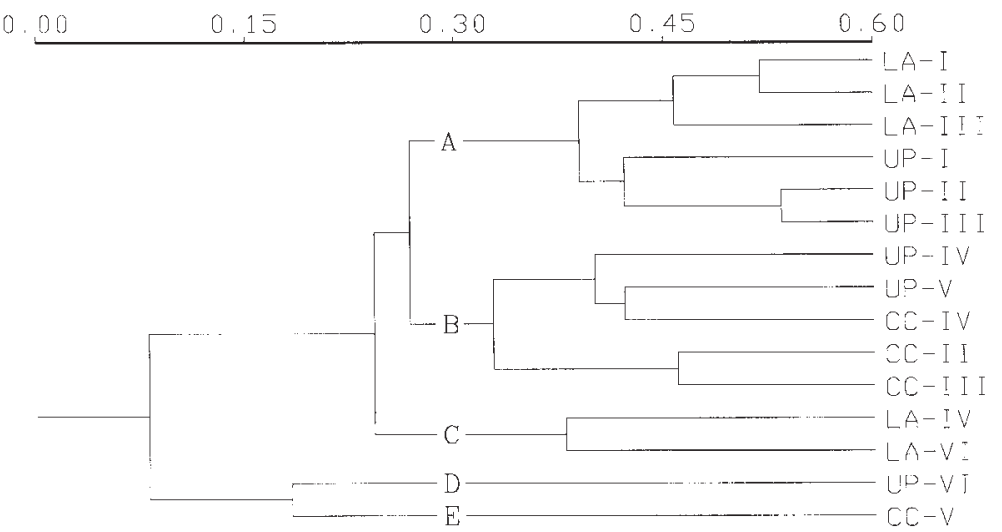

Fig. 3.- Clasificación de inventarios de acuerdo al índice de Sörensen y el método de agrupamiento UPGMA. Sectores biogeográficos (CC, UP y LA) y clases altitudinales (I a VI) como en la figura 2.

Fig. 3.- Classification of plots following the Sörensen index and the cluster analysis UPGMA. Biogeographic sectors (CC, UP and LA) and altitudinal levels (I a VI) as in figure 2.

que el inventario LA-VI (recuérdese que el LA-V, no existe) estuviese separado, como lo están UP-VI y CC-V. Esta composición puede ser en parte consecuencia de la existencia de una sola localidad en LA-VI (Laguna de Arbas) y de las características botánicas de ésta, muy parecidas a dos de las tres localidades de LA-IV (Puertos de la Magdalena y de Leitariegos, la otra de la clase es el Puerto de Ancares), estando situadas en el mismo subsector fitogeográfico, Laciano, y en el mismo piso, subalpino. Además el número de especies recogidas en cada una de esas cuatro localidades es relativamente parecido, contando con el enrarecimiento según se asciende: 23 en el Puerto de Ancares (la localidad más baja de todas ellas), 20 en el Puerto de Leitariegos, 14 en el Puerto de la Magdalena y 13 en la Laguna de Arbas. Ninguna de las cuatro especies exclusivas del grupo están presentes en el inventario LA-VI (Thelaxes suberi, especie mediterránea, y Calaphis betulicola son del Puerto de Ancares, Ctenocallis setosus del Puerto de la Magdalena y Subsaltusaphis lambersi del de Leitariegos). Además el número de especies en la única localidad de LA-VI (13) es muy superior a las dos que figuran en el inventario UP-VI (incluido en el grupo D) e incluso a las nueve del inventario CC-
V (grupo E). La separación de los dos últimos inventarios en sendos grupos se justifica en razón de su altitud, como ya se ha comentado.
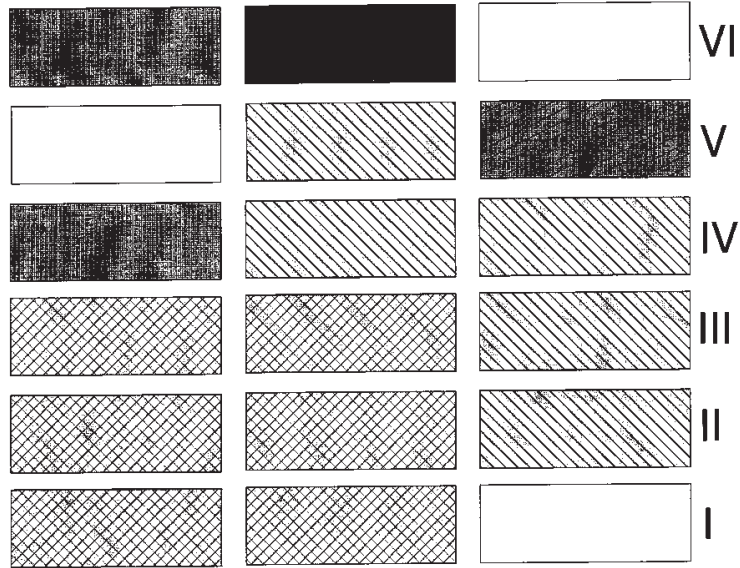

LA

UP

CC

Fig. 4.- Grupos de inventarios por sectores fitogeográficos y clases altitudinales (ver figura 3). En blanco: clases altitudinales no representadas en el sector fitogeográfico correspondiente.

Fig. 4.- Plot groups by phytogeographic sectors and altitudinal levels (see figure 3). In blanc: altitudinal levels not represented in the corresponding phytogeographic sector. 
A nuestro entender, el análisis de similitud efectuado no contradice lo expuesto en el epígrafe anterior sobre las clases altitudinales altas. De hecho los inventarios de las clases altitudinales IV a VI quedan reunidos, aunque en diferentes grupos, por lo expuesto y que no reiteramos.

La situación con respecto a lo expuesto sobre la clase I en dicho epígrafe es un poco diferente, pues ciertamente no quedan "aislados" sus inventarios (UP-I y LA-I). Pero, consideramos que la situacion es lógica si tenemos en cuenta el elevado número de especies comunes que presentan esos inventarios con los de las clases II y III, especialmente en el sector LA, pues UP-I está algo separado del conjunto UP-II y UP-III. En definitiva hay complementariedad en lo obtenido con ambos análisis, de diferente tipo.

\section{AGRADECIMIENTOS}

El estudio se ha realizado en el contexto de los proyectos de investigación "CAICYT 0141A.509/1985" sobre la entomofauna de la Provincia fitogeográfica Orocantábrica, "DGICYT PB-1987-0397, Fauna Ibérica I" y "DGICYT PB1989-0081, Fauna Ibérica II'. Expresamos nuestra gratitud a la Dirección General de Investigación Científica y Técnica del Ministerio de Educación y Ciencia de España por la financiación del programa de investigación en España de A.K. Ghosh. (SAB/93-0130). Dos de los autores (M.D. y N.N.) aprovechan la ocasión para agradecer al Prof. G. Remaudière (entonces, 1986, en el Institut Pasteur de París y actualmente en el Muséum National d'Histoire Naturelle de la misma ciudad) con quien compartieron muchas horas de campo en la montaña, todas sus enseñanzas, y al Prof. A. Penas Merino, Catedrático de Biología Vegetal de la Universidad de León, sus múltiples orientaciones y comentarios sobre temas botánicos y orocantábricos necesarios para el desarrollo de este trabajo.

\section{Referencias}

Dixon, A.F.G., 1985. Aphis Ecology. Blackie. Glasgow \& London. $157 \mathrm{pp}$

Ghosh, A.K. \& Nieto NAFría, J.M., 1994. Stratigraphic distribution of Aphidfauna (Hom.) in Eastern Andalusian Mountains (South Spain). Orsis, 9: 85-96.

GutiérRez Alaiz, I. \& Mier Durante, M.P., 1983. Chaitophorinae, Lachninae y Pterocommatinae de la provincia de León. Actas I Congreso Ibérico de Entomología, León: 329-338.
Hair, J.F., Anderson, R.E., Tatham, R.L. \& BLACH, W.C., 1992. Multivariate data analysis with readings. Macmillan Publishing Co.. New York. 544 pp.

ILHARCO, F.A., 1968. Algumas corecçoes e adiçoes à lista de afídeos de Portugal Continental. I Parte. Agro. Lus., 29: 117-139.

LEGENDRE, L. \& LeGENDRE, P., 1979. Ecologie numérique. 2: La estructure des donnés écologiques. Masson \& Presses de l'Université du Quebec. 247 pp.

LÓPEZ RodRÍGUEZ, R. \& NiETO NAFríA, J.M., 1983. Aportaciones al conocimiento de los Thelaxinae y Drepanosiphinae de la provincia de León (España). Boletín Asociación española Entomología, 7: 101-113

Nieto Nafría, J.M., Delfino, M.A. \& Mier Durante, M.P., 1994. La afidofauna de la Argentina: su conocimiento en 1992. Universidad de León, Secretariado de Publicaciones. León. 235 pp.

Nieto Nafría, J.M. \& Mier Durante, M.P., 1991. Caracterización morfológica y bionomía de Macrosiphum meixneri (Hom. Aphididae). Entomologia Generalis, 16(2): 123-131.

Nieto Nafría, J.M., Remaudière, G. \& Mier Durante, M.P., 1990. Newly recorded aphid species in the Phytogeographic Province Orocantabrian of Spain. Acta Phytopathologica Entomologica Hungarica, 25(1-4): 365-373.

Nieto Nafría, J.M., Remaudière, G. \& Mier Durante, M.P., 1991. Novedades para la afidofauna española en la Provincia fitogeográfica Orocantábrica (Cordillera Cantábrica, España). Boletín Asociación española Entomología, 15: 317-324.

NúÑEZ PÉREZ, E., 1992. Bases para el desarrollo del control integrado de los pulgones de los cultivos de la provincia de León. Universidad de León, Secretariado de Publicaciones. León. 7 pp. y 4 microfichas.

Rivas-Martínez, S., Díaz, T.E., Prieto, J.A.F., LOIDI, J. \& PENAS, A., 1984. La vegetación de la alta montaña cantábrica. Los Picos de Europa. Ediciones Leones. León. 300 pp.

Robles blanco, M.P. \& Mier Durante, M.P., 1982. Los géneros Macrosiphum, Sitobion, 
Uroleucon y Macrosiphoniella en la provincia de León (España). Boletín Real Sociedad española Historia Natural (Biología), 80(3-4) [1982]: 189-200.

Robles García, B \& Nieto NAFría, J.M., 1983. Algunos Aphidini de la provincia de León. Eos, 49: 255-268.

SNEATH, P.H.A. \& SOKAL, R.R., 1973. Numerical taxonomy. The principles and practice of numerical classification. W. Freeman. San Francisco. $573 \mathrm{pp}$.

Tizado Morales, E.J., 1991. Estudio comparado de la fauna y la biología de pulgones, afidíinos (Hym.,) y otros insectos acompañantes en dos áreas de la provincia de León. Univ. León (Secretariado Publicaciones): 8 pp. y 4 microfichas.
Tizado Morales, E.J. \& Nieto Nafría, J.M., 1991. Aportaciones a la afidofauna leonesa: novedades para la fauna española de la tribu Macrosiphini. Graellsia, 47: 43-48.

Tizado Morales, E.J. \& Nieto Nafría, J.M., 1994. A new species of Uroleucon (Hom. Aphididae) on Andryala spp.: a multivariate analysis. Canadian Entomologist, 126: 12511261.

Recibido, el 14-II-1993

Aceptado, el 5-VII-1995 Publicado, el 27-XII-1996 